This item was submitted to Loughborough's Research Repository by the author.

Items in Figshare are protected by copyright, with all rights reserved, unless otherwise indicated.

\title{
Turbulent jet characteristics for axisymmetric and serrated nozzles
}

\section{PLEASE CITE THE PUBLISHED VERSION}

http://dx.doi.org/10.1016/j.compfluid.2014.09.035

\section{PUBLISHER}

(c) Elsevier Ltd.

\section{VERSION}

AM (Accepted Manuscript)

\section{PUBLISHER STATEMENT}

This work is made available according to the conditions of the Creative Commons Attribution-NonCommercialNoDerivatives 4.0 International (CC BY-NC-ND 4.0) licence. Full details of this licence are available at: https://creativecommons.org/licenses/by-nc-nd/4.0/

\section{LICENCE}

CC BY-NC-ND 4.0

\section{REPOSITORY RECORD}

Xia, Hao. 2014. "Turbulent Jet Characteristics for Axisymmetric and Serrated Nozzles". Loughborough University. https://hdl.handle.net/2134/16449. 


\title{
Turbulent jet characteristics for axisymmetric and serrated nozzles
}

\author{
Hao Xia* \\ Thermo-Fluid Mechanics Research Centre, University of Sussex, Brighton BN1 9QT, UK
}

\begin{abstract}
Turbulent jet large eddy simulations (LES) are performed at Mach 0.9 and Reynolds number around $10^{6}$. Implicit large-eddy simulation (ILES) is employed, namely omitting explicit subgrid scale models. The Reynolds-averaged Navier-Stokes (RANS) solution is blended into the near wall region. This makes an overall hybrid LES-RANS approach. A Hamilton-Jacobi equation is applied to remove the disparate turbulence length scales implied by hybridization. Computations are contrasted for a baseline axisymmetric (round) nozzle and a serrated (or chevron) nozzle with high bending and penetration. Jet characteristics for both nozzles are studied in detail with well documented experimental data compared. The chevron effects are demonstrated by comparing both solutions using the same mesh resolution and flow conditions. Higher order velocity moments with potential for aeroacoustic modeling and noise prediction, such as the two-point velocity spatial correlations, are also explored. Numerical simulations presented in this study utilize an in-house flow solver with improved parallel scalability and efficiency by means of data packeting and a scheduling algorithm similar to the Round Robin scheduling.
\end{abstract}

Keywords: Large eddy simulation, turbulent jet, serrated nozzle, message passing interface, scheduling

\section{Introduction}

Jet dynamics has long been a key topic in fluid dynamics due to its high theoretic importance in understanding turbulence, but also because of the impact on understanding and controlling jet noise. The axisymmetric jet (or round jet) represents a benchmark for research into the physics of fluid flow. This is evidenced by the volumes of classical publications involving experimental data,

\footnotetext{
*Email: h.xia@lboro.ac.uk. Present address: Department of Aeronautical and Automotive Engineering, Loughborough University, Loughborough LE11 3TU, UK.
} 
mathematical analysis, and computational modeling [17, 10, 30, 20]. In aeroacoustics, jet noise is the subject that focuses on the noise generation caused by high-velocity jets and the turbulent eddies formed by shearing flow. While the fundamental physics of turbulence remains an undisputedly challenging topic, the latter jet noise however has much closer relation with industrial applications. Jet noise has been widely studied since the 1950s when Lighthill $[14,15]$ first postulated an acoustic analogy which was originally aimed to separate the generation and prorogation of sound through mathematical manipulation of the Navier-Stokes equations. Since then, most modeling work has mainly been analytical until recent advances in computational aeroacoustics (see for example Bodony and Lele's review [2]).

Noise reduction nozzles are of great interest to the aerospace industry, such as the serrated (or chevron) nozzles [3]. Their potential is promising - as a matter of fact some designs are already in service [1]. As shown in the experiments by Saiyed et al. [22, 23], serration modification to the round nozzle can bring as much as $3 \mathrm{~dB}$ reduction in peak noise during take-off with less than $0.5 \%$ thrust loss during cruise. For high frequencies and large angles to the jet, the use of chevrons may also lead to about to $2 \mathrm{~dB}$ noise increase. This naturally leads to the nozzle design optimistion problem in which eddy resolving numerical simulations and acoustic modeling techniques for jet noise prediction potentially play an important role.

The desire to more reliably predict the noise reduction available to individual jet nozzle design concepts has led to the introduction of large-eddy simulation (LES) techniques to jet noise problems, of course thanks to the enormous advances in computer power over the last 2 to 3 decades. The noise radiated from jet exhaust (historically termed "jet noise") has received significant attention. For example, Xia et al. [38, 37] recently made attempts to predict far-field noise radiated from chevron nozzles, Uzun and Hussuni [34] showed high frequency spectra of a chevron jet, while Shur et al. [25, 24] explored a generic approach for emulating complex nozzle jets. In order to understand noise generation (reduction) mechanisms and to potentially improve prediction, the turbulent characteristics of the jet flow, especially for complex nozzle geometry, need to be explored in contrast to a baseline axisymmetric nozzle.

In this study, turbulent characteristics for a round and serrated nozzle from the experimental study by Bridges and Brown [3] are compared by means of numerical simulation (geometry shown in Figure 1). The aim is to assess the sensibility of the numerical methods and the flow solver due to the alteration of nozzle geometry . This assessment is of particular interest if they are to be used 


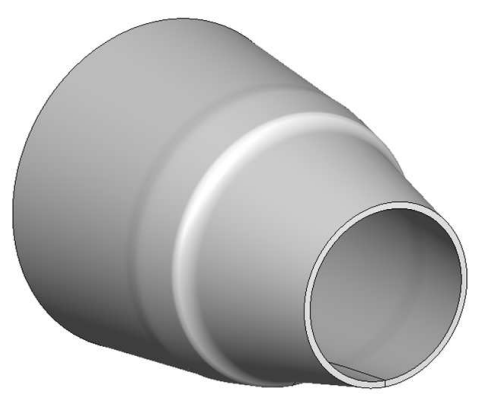

(a) Baseline

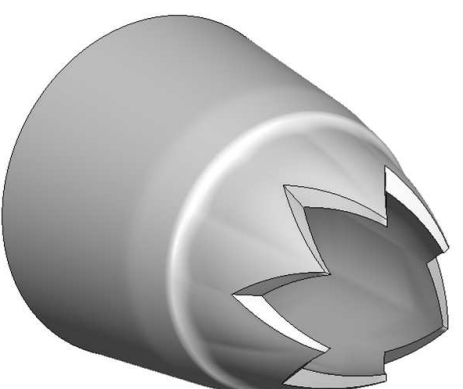

(b) Serrated

Figure 1: Nozzle geometry by NASA Glenn for: (a) the baseline round nozzle SMC000; and (b) the serrated nozzle SMC006

for more design oriented tasks in the future. The paper is organized as follows. First, $\S 2$ Numerical Methods gives a brief description of the governing equations, discretization schemes, and LESRANS hybridization. In $\S 3$ Results and Discussion, the simulation setup and flow conditions are briefly given. Results are further discussed and compared for the axisymmetric and serrated nozzle, respectively, where jet characteristics are explored by presenting centerline velocities/Reynolds normal stress $u_{r m s}^{\prime}$, velocity second moment radial profiles, and two-point velocity correlations. The instantaneous growth of the shear layer is also compared between two nozzles. Finally, in $\S 4$ we discuss the potential advantages of a user-level Round Robin type scheduling (rather than the network scheduling at the MPI level) for communications among different mesh partitions.

\section{Numerical methods}

The Favre-average/filtered compressible Navier-Stokes equations for ideal gases are solved with a finite volume in-house solver. When an adverse pressure gradient is present at the nozzle wall boundary, a laminar type boundary velocity profile is prone to separate non-physically, dramatically altering the subsequent downstream jet development. Naturally, unless fully resolved, the boundary layer needs some kind of treatment, where RANS seems highly suitable among many other options. More importantly a proper turbulent boundary layer mean velocity profile is crucial to jet shear layer development downstream. The hybrid LES-RANS approach [32, 31] with a Hamilton-Jacobi equation blending the RANS and LES zones has recently been successfully applied by the author and coworkers to chevron jets [38], and is adopted in the present study. In the near wall regions the Spalart-Allmaras RANS model [27] is applied. The wall distance is efficiently computed using 
a level set type approach by Tucker [31] and by Xia and Tucker [36]. Based on the modified wall distance $\tilde{d}$, the RANS and LES regions are defined and blended by a Hamilton-Jacobi type equation:

$$
\sqrt{\left(\frac{\partial \tilde{d}}{\partial x}\right)^{2}+\left(\frac{\partial \tilde{d}}{\partial y}\right)^{2}+\left(\frac{\partial \tilde{d}}{\partial z}\right)^{2}}=1+f(\tilde{d})\left(\frac{\partial^{2} \tilde{d}}{\partial x^{2}}+\frac{\partial^{2} \tilde{d}}{\partial y^{2}}+\frac{\partial^{2} \tilde{d}}{\partial z^{2}}\right)+g(\tilde{d})
$$

where $f(\tilde{d})=\epsilon_{0} \tilde{d}, g(\tilde{d})=\epsilon_{1}\left(\tilde{d} / d_{c}\right)^{2}$. $\quad d_{c}$ is the RANS cut-off distance corresponding to nondimensional wall unit $y^{+} \approx 60$. Notice the above equation is solved as an auxiliary equation to the main flow equations, which is intrinsically parallel and reduces the cost of a crude search for the nearest wall distance. This formula essentially leads to $\tilde{d}=0$ on the wall and in the off wall LES region, where the production of eddy viscosity is therefore also zero, as no explicit subgrid scale modeling is used. The smooth transition of the eddy viscosity field between the RANS and implicit LES zones seems more reasonable and helps maintain good numerical stability. Also, as outlined in [33], smoother fields give potentially improved RANS model behavior around singularities and convex corners.

For the inviscid flux at a control volume common face, the MUSCL reconstruction [35] with a modified Roe's scheme [21] is used to limit the excessive amount of dissipation due to upwinding:

$$
\mathbf{F}=\frac{1}{2}\left(\mathbf{F}_{L}+\mathbf{F}_{R}\right)-\epsilon \frac{1}{2}\left(\mathbf{Q}_{R}-\mathbf{Q}_{L}\right)
$$

where $\mathbf{Q}$ and $\mathbf{F}$ are the conservative and flux vectors respectively, and the smoothly varying parameter $0.1<\epsilon<1$ is defined according to Xia et al. [38]. Similar techniques have also been successfully explored by Bui [6], Mary and Sagaut [16] and Ciardi et al. [7].

For time advancing, the dual-time scheme is employed with the outer physical time discretized with a three-level backward Euler scheme leading to second-order temporal accuracy. The inner pseudo time is advanced using a three-stage low storage Runge-Kutta scheme. The implicit outer time discretization also means relatively larger physical time steps are allowed, free from limitations due to locally small grid elements.

\section{Results and discussion}

\subsection{Computational overview}

The cold jet flow conditions (Test Point 7 of Tanna [29]) are specified. These conditions are widely used in jet dynamics and noise experiments with an acoustic Mach number at the jet 
exit $M a_{a c}=U_{j} / a_{\infty}=0.9$ and a temperature ratio $T_{j} / T_{\infty}=0.84$. The ambient conditions are $p_{\infty}=0.97 \times 10^{5} \mathrm{~Pa}$ and $T_{\infty}=280.2 \mathrm{~K}$. Reynolds number is around $10^{6}$ based on the nozzle exit diameter $D$ and jet exit velocity $U_{j}$.

The axisymmetric nozzle SMC000 has a 2-inch exit diameter while the serrated SMC006, although deviated from SMC000, has a slightly reduced effective jet diameter due to the inward bending of the chevrons. SMC006 is serrated equally in the circumferential direction with six chevron tips and six notches with each chevron corresponding to a $\pi / 3$ sector, and is placed in a position so that planes $z=0, y=0$ cut right through a pair of tips and notches, respectively.

Solutions are obtained on grids ranging from 7 to 20 million grid points. A singularity treatment is needed for the jet centerline thus avoiding clustering polar points or polar lines. Previous studies show that for SMC006 the flow appears to be strongly non-parallel with high values of mean radial velocity in the near nozzle region. The finner grid features doubled azimuthal grid points (240 in total) and faster radial spreading, with lip line radius doubled from $x=0$ to $1.5 D$, followed by a slower spreading - this apparently is in accord with the jet shear layer growth. The LES domain comprises non-reflective BCs in the far-field and "sponge" zones with ramped numerical dissipation towards the downstream boundary. The wall units near the nozzle exit boundary are controlled such that $\Delta r^{+} \approx 2.5, \Delta x^{+} \leq 300$ and $\Delta(r \theta)^{+} \leq 30$. Physical time step is set to be one thousandth flow through time, $10^{-3} D / U_{j}$. In practice, to reach a well developed jet $100 \sim 200$ thousand physical time steps are normally needed and another $100 \sim 300$ thousand are further advanced to obtain turbulent statistics. For higher order moments, such as the two-point spatialtime cross correlations, 300 thousand steps are found to be required. Details of the running cases are summarized in Table 1.

Table 1: Numerical simulation case summary.

\begin{tabular}{cccccc}
\hline Case ID & Nozzle & Serrations & Grid points & Run time: $\times D / U_{j}$ & CPU cores $\times$ hours \\
\hline R007M & SMC000 & n/a & $6,450,227$ & $(200+300)$ & $72 \times 1300$ \\
R020M & SMC000 & n/a & $20,792,017$ & $(200+200)$ & $512 \times 550$ \\
S620M & SMC006 & 6 & $20,792,017$ & $(200+200)$ & $512 \times 550$ \\
\hline
\end{tabular}




\subsection{Axisymmetric}

Axisymmetric jet has been extensively studied experimentally, analytically and numerically in the last 50 years. There is a host of literature available on the experiments which can be used to validate our numerical simulations. For instance, Moore [18] measured subsonic turbulent jets under the influence of instability waves and their effects on jet exhaust noise generation, Lau et al. [13] measured the dynamics of both subsonic and supersonic free jets, Panchapakesan and Lumley [19] studied the second and third velocity moments of a turbulent round air jet, Hussein et al. [11] measured high Reynolds number jets to assess their momentum-conserving and self-similarity features, and Arakeri et al. [9] discovered the use of micro transverse jets can have effects on noise reduction of the main jet. The list could go on, and the resent report of Bridges and Wernet [5] summaries a series of NASA jet experiments. It is fair to say these aforementioned experiments are sufficient to provide validation databases for numerical simulations.

Mean velocity and Reynolds normal stress on the centerline are shown in Figure 2. Figure 2 a compares the decaying centerline mean axial velocity. Symbols are measurement data from Refs. [18, 13, 9, 5]. Results from two numerical simulations, R007M and R020M, are also presented with the former on a grid with $7 \mathrm{M}$ nodes and the latter $20 \mathrm{M}$ nodes. The length of potential core varies slightly from R007M $(\sim 5.5 D)$ to R020M $(\sim 6.5 D)$. Similarly, different measurements also show this difference with Moore's potential core length being the shortest. Overall, the agreement between numerical predictions and experiments are encouraging, especially in the " $1 / x$ " decaying range.

Figure $2 \mathrm{~b}$ plots the inverse of Figure 2a. Experiments on high-Reynolds-number fully developed jets have shown that the centerline mean velocity, $U_{c}$, decays as

$$
\frac{U_{c}}{U_{j}}=B_{u}\left(\frac{D}{x-x_{0}}\right)
$$

where $x_{0}$ is the axial coordinate of the virtual origin of the jet and the constant $B_{u}$ is approximately 5.8 for the experiments of Hussein et al. [11]. Without any adjustment of the potential core length, our predictions show a virtual origin of $x_{0}=0.73 D$ and $0.24 D$ for R007M and R020M, respectively. The corresponding $B_{u}$ values are 6.6 and 7.9. These are highlighted by the doted and dash dot straight lines in Figure 2b. Because the inverse, $U_{j} / U_{c}$, is a straight line in the decaying range, many choose to plot the inverse to highlight the slope $B_{u}$ and the virtual origin $x_{0}$ (the intersection with $x$-axis). 


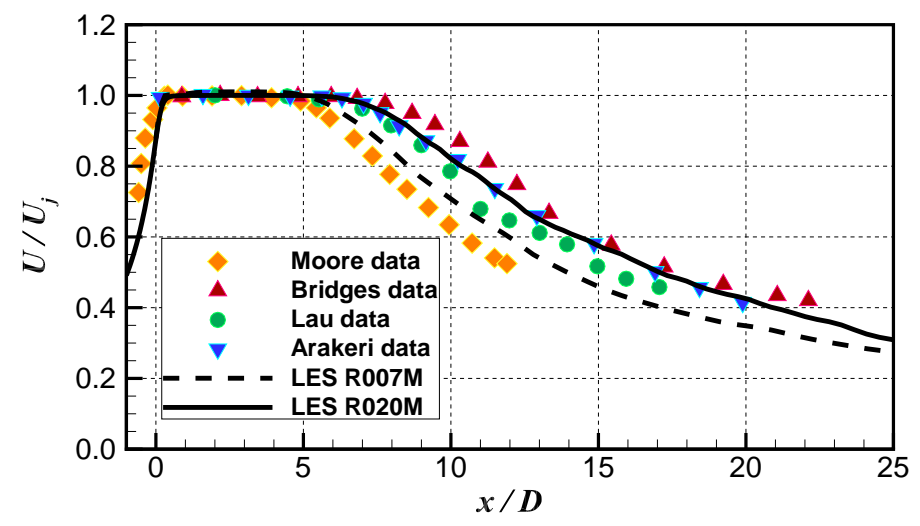

(a) Decaying mean velocity

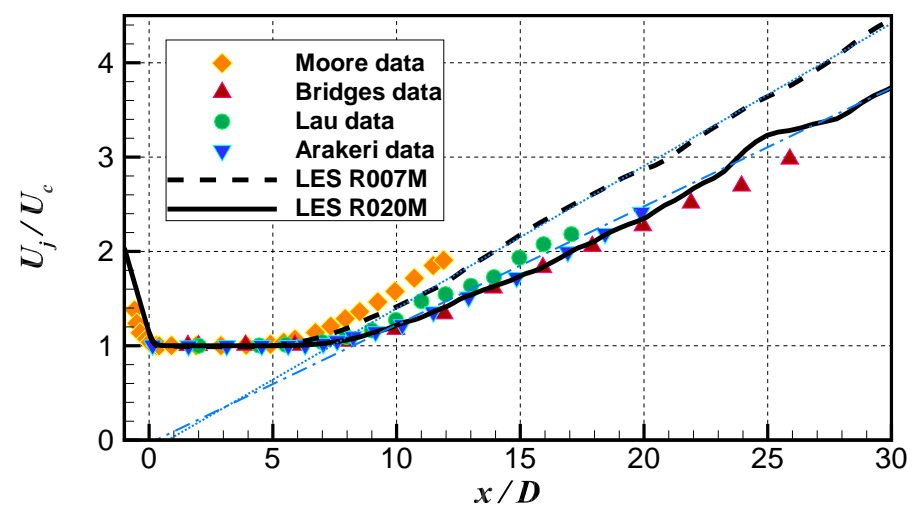

(b) Virtual origin

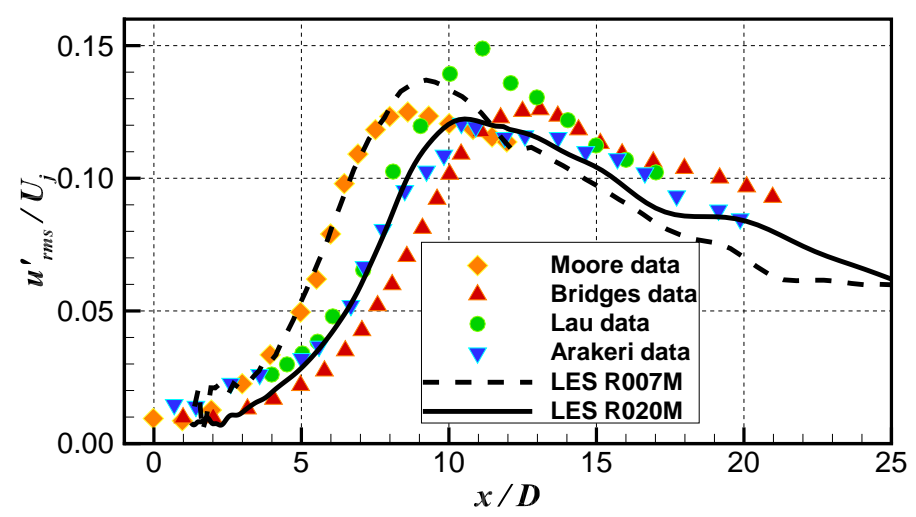

(c) Reynolds normal stress

Figure 2: Centerline characteristics of the baseline round jet SMC000, present predictions compared with measurements $[18,13,9,5]$. 
The normal Reynolds stress component $\overline{u^{\prime} u^{\prime}}$ is shown in Figure 2c, which is more conveniently expressed as $u_{r m s}^{\prime} / U_{j}$. Like the mean velocity, the normal stress peaks earlier and higher for the coarser grid solution. However, that is within the range of the measurement difference. More importantly, the growth and decay of the Reynolds stress are in good agreement with the experiments. The laser velocimetery (LDV) measurement of Lau et al. [13] peaks considerably higher (about $20 \%$ ) than other particle image velocimetry (PIV) measurements and the numerical prediction. These unusually higher peak values due to the underlying experimental techniques were addressed at some length by Lau et al. [13] and Arakeri et al. [9].

Concerning Reynolds stresses, we now discuss the apparent self-similar behavior of jet dynamics and compare to measurement data in Figure 3. Favorable agreement is obtained for all three second moments of velocity when $x \geq 15 D$. The simulation data collapses reasonably to a single curve for various $x$-locations, and their match with the air jet data of Panchapakesan and Lumley [19] is encouraging, except for normal stresses $\left\langle u^{\prime} u^{\prime}\right\rangle$ and $\left\langle v^{\prime} v^{\prime}\right\rangle$ when $r \rightarrow 0$. Shear stress data agrees particularly well across the jet shear layer for the given $x$-locations. Notice $\langle\cdots\rangle$ is used for the ensemble average, a combination of time- and spatial-average, which is different from the centerline quantities where only the time-average (denated by $\cdots$ ) is possible.

The two-point velocity spatial transverse correlations without any time delay defined as

$$
R_{11}(\Delta y)=\frac{\overline{u^{\prime}(x, y, z, t) u^{\prime}(x, y+\Delta y, z, t)}}{\overline{u^{\prime}(x, y, z, t) u^{\prime}(x, y, z, t)}}
$$

are shown in Figure 4 for base location: $x=16 D, y=0$ and $z=0$. The transverse offset $\Delta y$ varies between $-2.5 D$ and $2.5 D$. Both correlations agree favorably, decay rapidly away from the center and reach below zero at $\Delta y \approx \pm 0.6 D$ indicating the jet is fully turbulent.

\subsection{Serrated}

Ensemble averaging is applied to obtain mean quantities. Since the SMC006 nozzle itself is azimuthally periodic (every $\pi / 3$ ), periodic average in the azimuthal direction can be further applied. Figure 5a compares the centerline mean axial velocity decay for LES R020M and LES S620M against the NASA SHJAR measurement data [5, 3, 38], where the differences of numerical predictions and measurements are shown to be within 1-2\%.

For the centerline normal Reynolds stress $u_{r m s}^{\prime}$, the simulations are, on average, within $5 \%$ of the measurements, but also experiencing some oscillation near the nozzle $(x<2 D)$ and in the downstream $(x>15 D)$. For the baseline round jet, the end of potential core is normally 


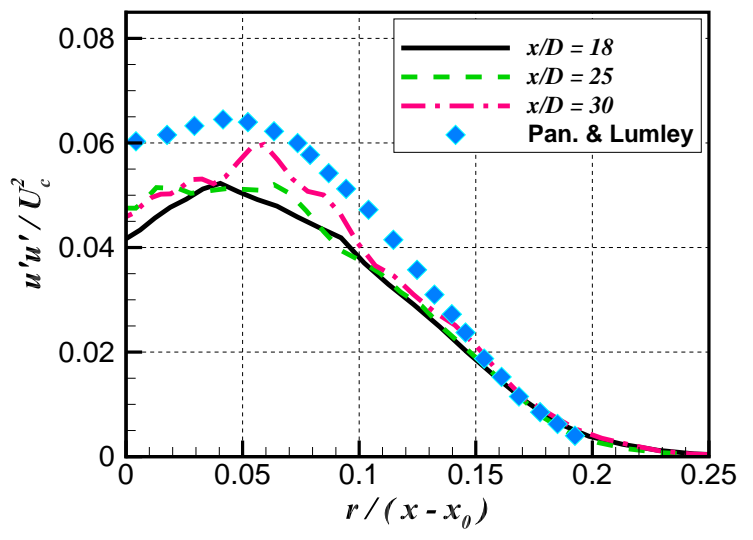

(a) $\left\langle u^{\prime} u^{\prime}\right\rangle / U_{c}^{2}$

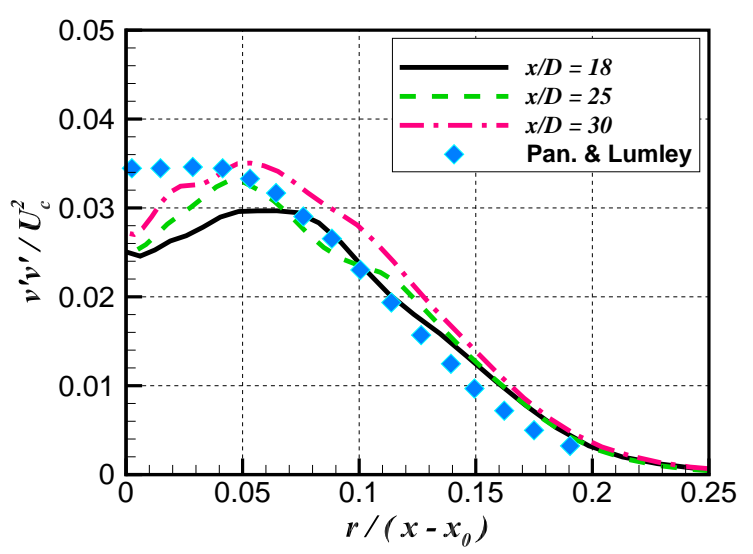

(b) $\left\langle v^{\prime} v^{\prime}\right\rangle / U_{c}^{2}$

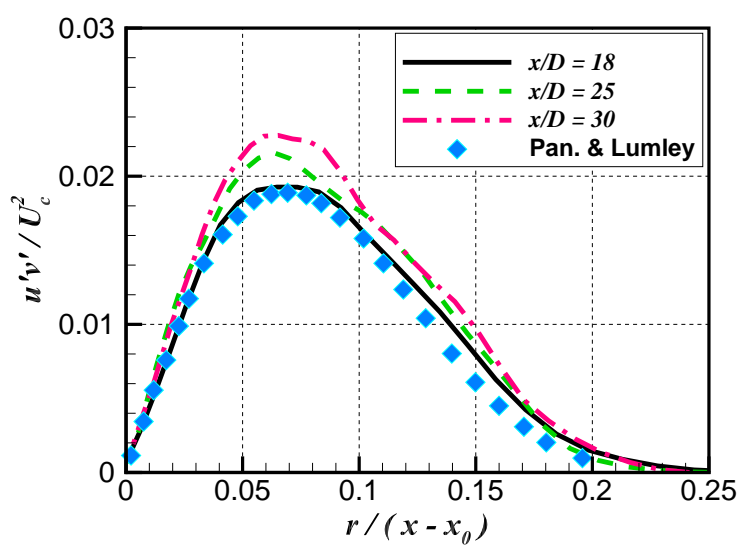

(c) $\left\langle u^{\prime} v^{\prime}\right\rangle / U_{c}^{2}$

Figure 3: Second moments of velocity across the jet shear layer at axial locations: $x=18,25,30$ (lines) and measurements (symbol) [19]. 


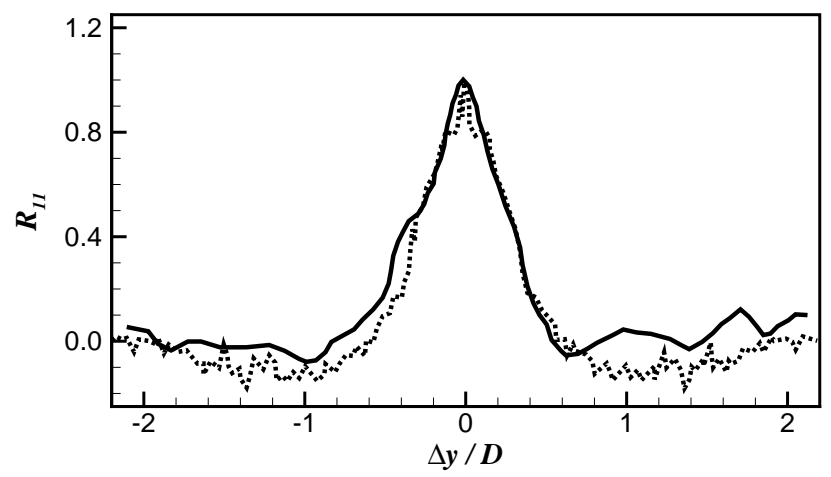

Figure 4: Two-point velocity spatial correlation $R_{11}$ at $x / D=16, y=0 . \cdots$, , measurement [4]; - , prediction.

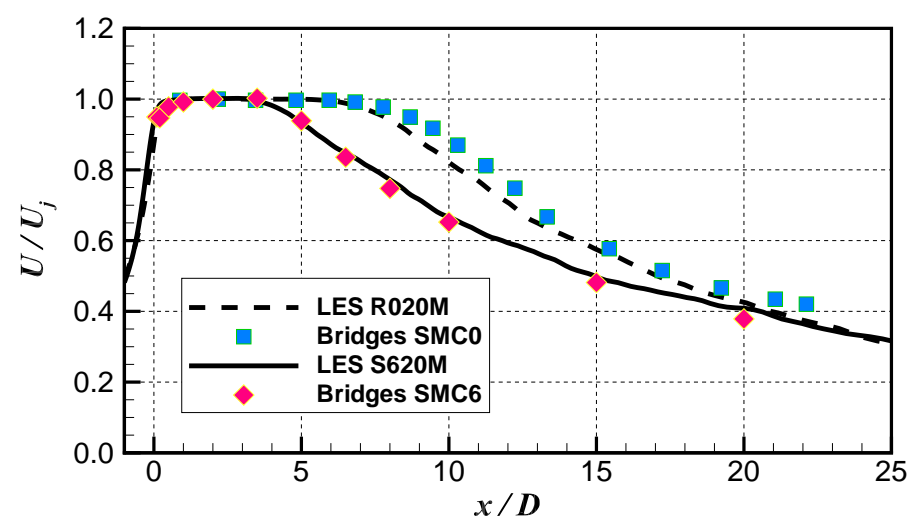

(a) Decaying mean velocity

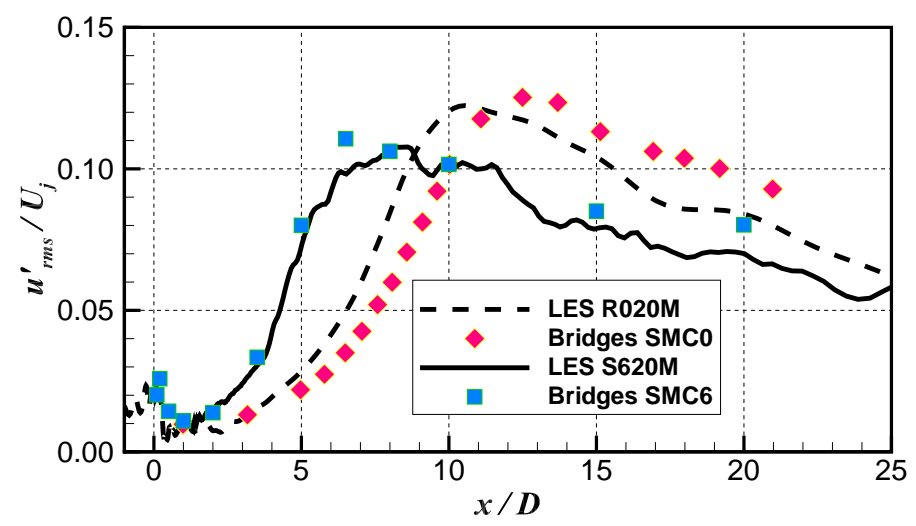

(b) Reynolds normal stress

Figure 5: Centerline characteristics of the serrated jet SMC006 and the baseline round jet compared to measurements $[3,5]$. 
around $x=6.5 D$. This can often be verified by the location where the centerline velocity starts to decay and the Reynolds stress peaks. For the serrated SMC006 nozzle, the potential core is notably shortened, $\sim 3.5 D$, which is considerably shorter than that of SMC000. This again can be interpreted as the result of the enhanced mixing dispersing the momentum of the core jet stream caused by the much increased radial velocity near the exit. The indication is that the flow past tips tends to go inward whereas the flow through the notches outward creating extra streamwise vorticity, hence more mixing.

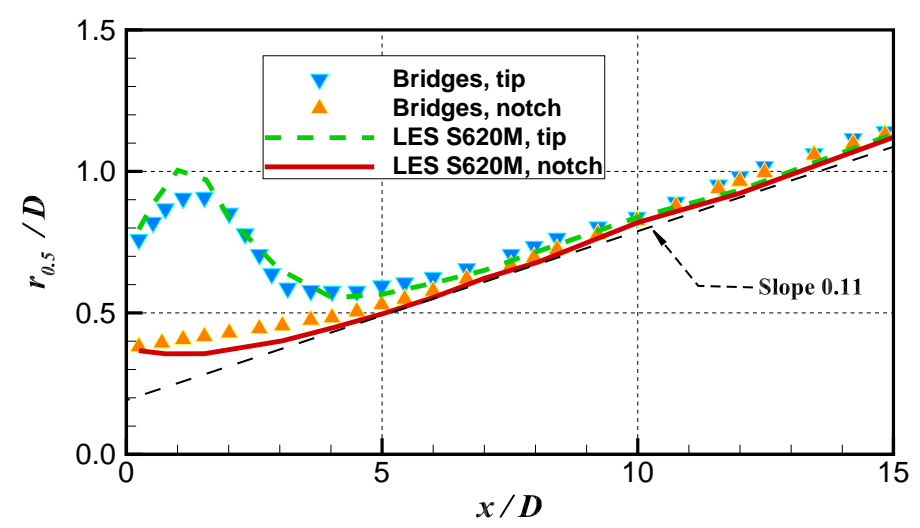

Figure 6: Comparison of the jet spreading rate depicted by the half-velocity jet width. Lines, LES S620M prediction; Symbols, measurements [3, 38].

Figure 6 shows that linear growth of the half-velocity jet width $r_{0.5}$. The half-velocity jet width reveals the geometric development of the jet shear layer. It is defined as the distance measured between the centerline and a point in the shear layer where the local mean velocity is equal to half of the local centerline or maximum mean velocity, namely $\left\langle u\left(r_{0.5}\right)\right\rangle=0.5 U_{c}$. Encouraging agreement between the present LES and the measurement is obtained. One can only distinguish the tip profile and notch profile for $0<x / D<6$. Strong serration effect takes place within the first 5 jet diameters. Towards the end of potential core and further downstream, the jet tends to be statistically axisymmetric. This linear behavior in the downstream can be even quantified by plotting the auxiliary dash line (also shown in Figure 6), where a linear fit yields $r_{0.5}=0.11 x-0.0072$. This is close to the "universal" round jet spreading rate as described by Davidson [8].

As discussed previously, two-point correlation without time delay plays a role in indicating turbulence length scale and the flow generally being laminar or turbulent. Now we further show 


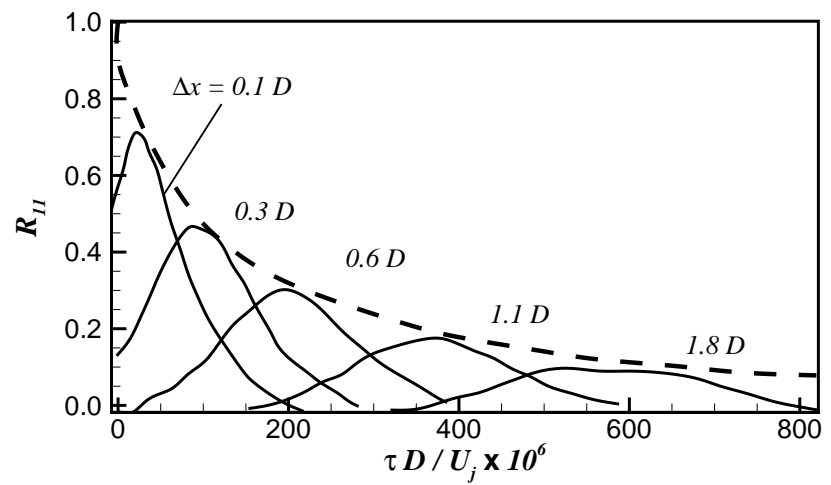

Figure 7: Two-point velocity longitudinal correlations with spatial separation $\Delta x$ and time delay $\tau D / U_{j} \times 10^{6}$. LES S620M; ---, envelop curve (corresponding to the auto-correlation).

the two-point velocity correlations with both spatial separation and time delay, which particularly has importance for jet noise modeling such as the well-known Tam \& Auriault model [28]. Figure 7 shows the spatially separated and temporally delayed longitudinal $R_{11}$ for SMC006:

$$
R_{11}(\Delta x, \tau)=\frac{\overline{u^{\prime}(x, y, z, t) u^{\prime}(x+\Delta x, y, z, t+\tau)}}{\overline{u^{\prime}(x, y, z, t) u^{\prime}(x, y, z, t)}}
$$

This also helps understand differences in integral scales and eddy convection speed. The reference probing point is fixed at $x=2 D$ and $y=0.5 D$ and on a "tip-cut" plane (e.g. $z=0$ ). All correlations are normalized by the auto-correlation of each signal at zero time delay. Notice that the envelope curve of the $R_{11}$ for different $\Delta x$ is indeed the auto-correlation at the base point and $R_{11}$ distributions exhibit a typical Gaussian profile.

In Figure 8 we further show the comparison of the shear layer development for SMC000 and SMC006. Depicted by instantaneous vorticity contours, the $z=0$ cut plane is shown for both R020M and S620M (a pair of notches in this case). The dimensional vorticity magnitude $|\boldsymbol{\Omega}|=$ $|\nabla \times \mathbf{v}|$ is ranging from 2,000 through 150,000 (or 0.85 to 25 in non-dimensional values). It is evident that the core of the shear layer has a much different development patten. The serrated case presents a much thicker (almost doubling the axisymmetric case) layer up to the end of the potential core and the initial flow direction is almost 30 degree outward, whereas in the round jet case the shear layer has a streamwise change dominant nature. 


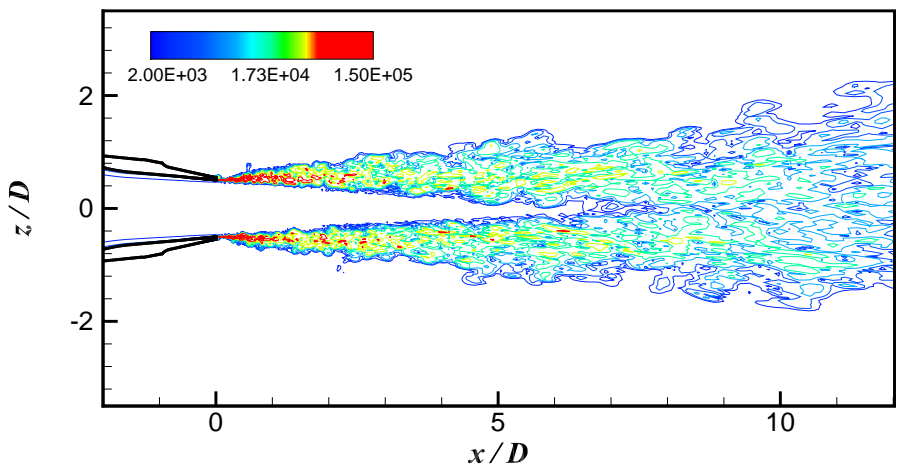

(a) Baseline SMC000

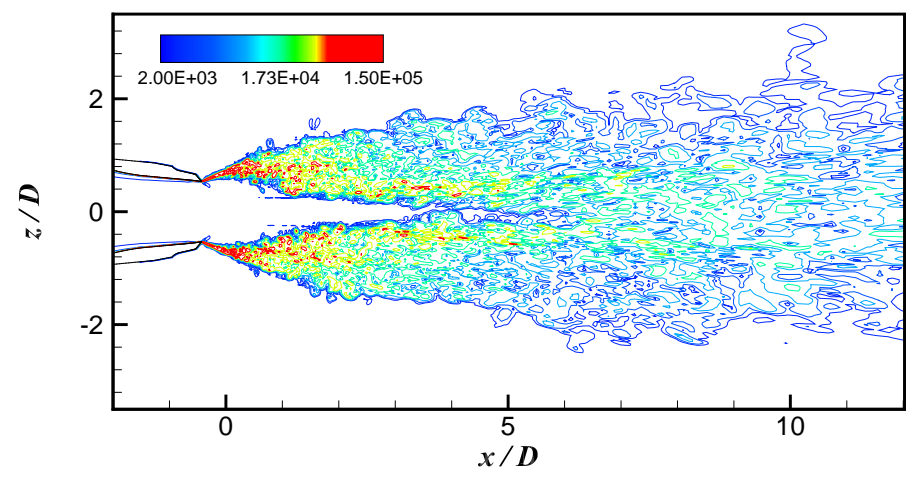

(b) Serrated SMC006

Figure 8: Shear layer growth depicted by instantaneous vorticity contours, both plots on the $x-z$ cut plane at $y=0$ : (a) baseline round; (b) serrated (cut plane through a pair of notches). 


\section{Improved scalability with user-level scheduling}

The parallelism of the in-house code used in this study is based on the use of the graph partitioning algorithm (METIS) outlined in LaSalle and Karypis [12]. The aim is to achieve minimum communication between different partitions as well as load balance. The parallelism is implemented using Message Passing Interface (MPI). Due to the limited capacity of the interconnect between different computing nodes in a typical HPC cluster, scheduling the message passing at user-level becomes important. MPI has its own low-level non-blocking mechanism to sequence these messages at the API-level. However, excessive non-blocking calls can eventually exhaust the buffer memory of the MPI layer. In our experience, this is found to be the case for relatively large cases.

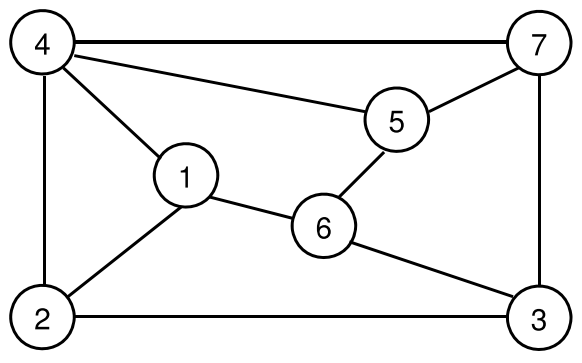

(a) Graph

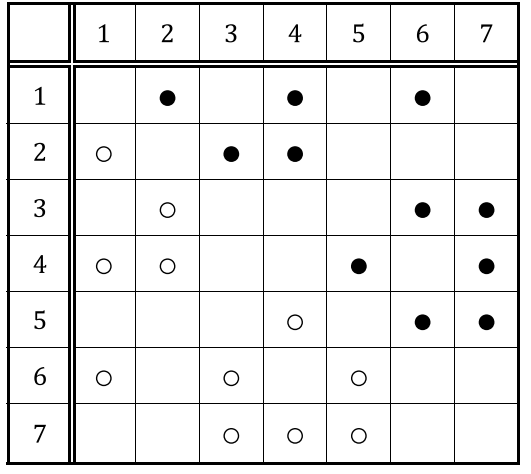

(b) Connectivity matrix

Figure 9: A 7-partition grid with inter-partition communication shown in the graph and connectivity matrix.

A simplified example can be used to demonstrate the potential benefit of a user-level scheduling. Figure 9a shows a 7-partition grid, where data communication between different partitions is presented in the graph. The corresponding connectivity is also shown in the table of Figure 9b. Because data exchange between any two partitions is symmetric (or non-directional since the same amount of information goes both ways), there are total 11 conversations (message passings). We only need to count the filled circles in Figure 9. The open circles are the transpose of the filled ones. We further assume the following two conditions are generally satisfied:

$C 1$ : Data exchange size between any two partitions is assumed to equal among all partitions.

C2: No partition appears more than once during the same round. 
Although the METIS algorithm of LaSalle and Karypis does not guarantee equal communication among all partitions, it produces in general reasonably similar sizes of partition facets between partitions while maintaining load balance and minimum overall communication. Therefore, assuming $C 1$ is accepted as a first step and is only discussed in this study. Taking into account non-equal communication would lead to the use of weighted graphs which will be addressed in the future work. Evidently, better parallel scalability/efficiency strongly depends on the total number of communication rounds. The fewer total rounds the better. Hence, $C 2$ is naturally satisfied, otherwise multiple appearances of the same partition in one round would cause significant waiting time for this partition and effectively all other partitions as well.

\begin{tabular}{llll|llll} 
R1: & $1-2$ & $3-6$ & $4-5$ & R1: & $1-2$ & $3-6$ & $4-5$ \\
R2: & $1-4$ & $2-3$ & $5-6$ & R2: & $1-4$ & $2-3$ & $5-7$ \\
R3: & $1-6$ & $2-4$ & $3-7$ & R3: & $1-6$ & $2-4$ & $3-7$ \\
R4: & $4-7$ & & & R4: & $4-7$ & $5-6$ & \\
R5: & $5-7$ & & & & & &
\end{tabular}

Figure 10: A sequential scheduling of five rounds of communication (left) versus the optimal scheduling of four rounds (right).

Figure 10 presents two different schedulings for the example case. On the left is a sequential one, which is obtained by sequentially walking through each row of the connectivity matrix (only upper triangle) in Figure 9b. The construction of a sequential round table is outlined in Alogrithm 1. It can be easily noticed that the sequential scheduling in this case is not optimal, especially both R4 and R5 involving only Partition-7. An optimal scheduling can be achieved by simply swapping communication 5-6 and 5-7 and subsequently moving 5-6 to R4. This results in a total number of 4 rounds as opposed to the sequential 5 rounds giving $25 \%$ improvement in overall communication speed.

For small size problems, an exhaustive list of round tables can be constructed in order to find the optimal scheduling. To some extent, this is similar to the so-called Round Robin scheduling [26] procedure to determine a sequence that ensures the total number of non-overlapping communication rounds is at its minimum, resembling seeking minimum rounds of matches, say, in a football tournament.

For larger size problems (virtually any practical problems with total communications exceeding 


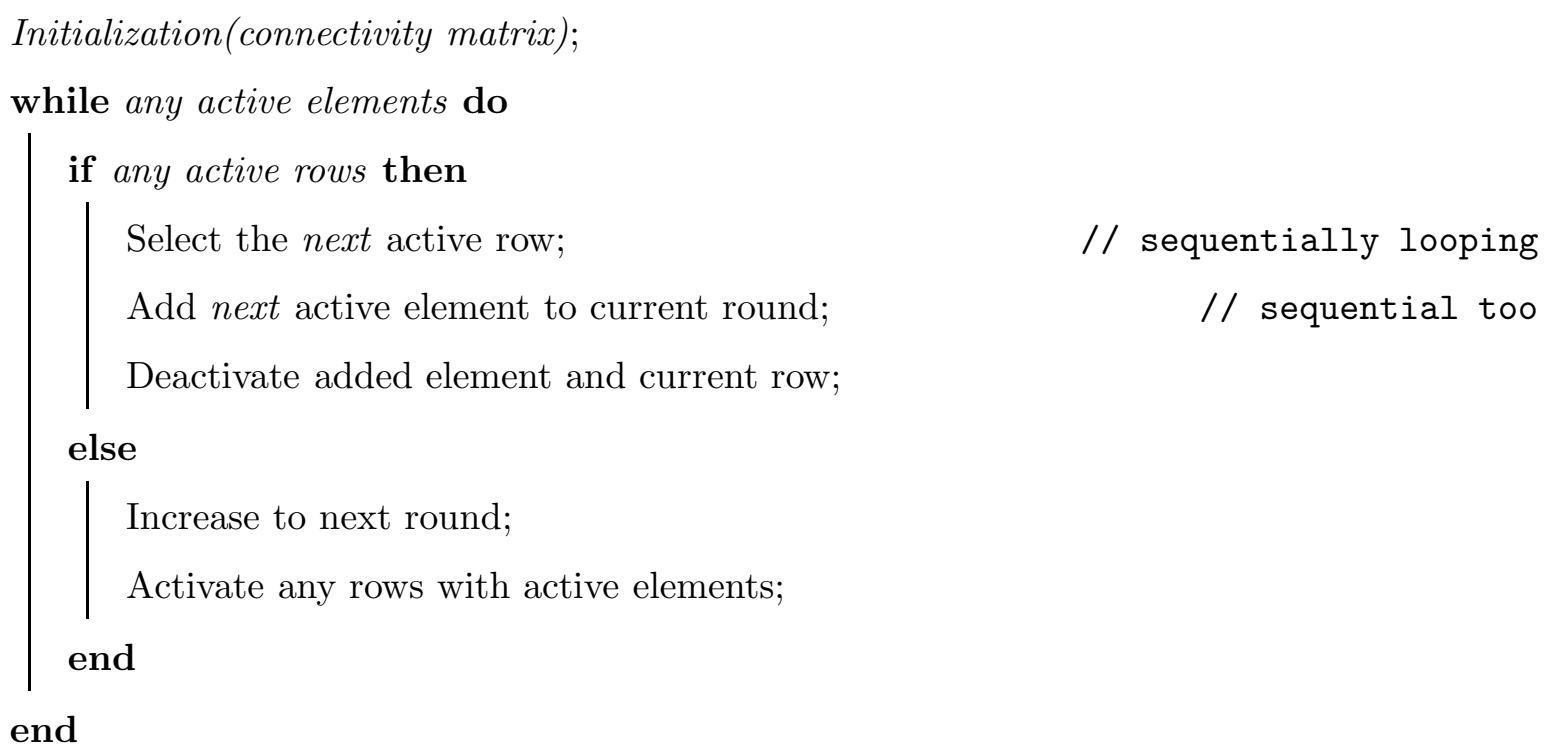

Algorithm 1: Construction of a sequential round table

Initialization(connectivity matrix);

while any active elements do

if any active rows then

Randomly select the next active row;

// from all active rows

Add a random active element to current round;

// from the selected row

Deactivate added element and current row;

else

Increase to next round;

Activate any rows with active elements;

end

end

Algorithm 2: Construction of round table in a "random" order 
20), however, it is prohibitive to create an exhaustive list of round tables in order to find the minimum rounds. Instead, a "random" sampling approach is adopted, in which the active rows and elements from the connectivity matrix are selected randomly rather than sequentially. To produce good sampling, the typical sampling size should be at least 10,000. Once the sampling round tables are obtained, it is straightforward to find the minimum rounds by comparison. It is noted that in this random sampling approach the obtained minimum number of rounds is not guaranteed to be the theoretical optimal value, rather, it can only be regarded as the best among the samples.

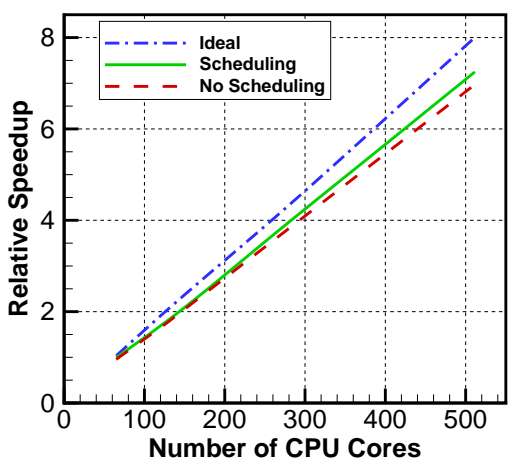

(a)

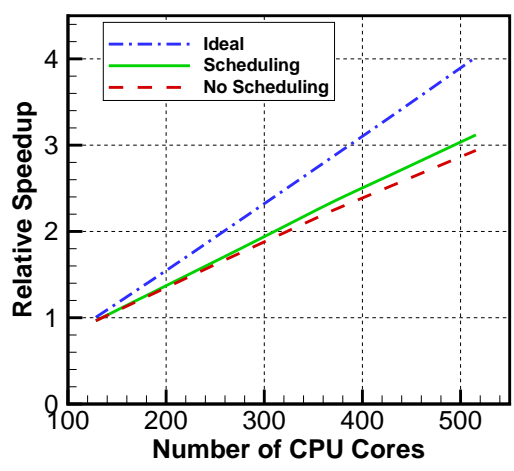

(b)

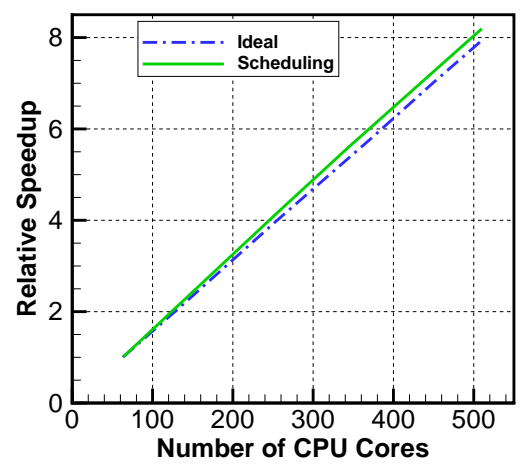

(c)

Figure 11: Parallel performances on PRACE supercomputers: (a) 64 to 512 cores on HERMIT (strong scaling); (b) 128 to 512 cores on CURIE (strong scaling); (c) 64 to 512 cores on CURIES (weak scaling).

Scalability test results are shown in Figures 11. In the context of high performance computing there are two commonly used notions for scalability, namely the strong and weak scaling. The strong scaling is defined as how the solution time varies with the number of processors for a fixed total problem size (e.g. grid points), whereas the weak scaling defined as how the solution time varies with the number of processors for a fixed problem size per processor.

For strong scalability, our mesh size is fixed at 20 million and the number of CPU cores varies. The relative speedup for the strong scaling is defined as:

$$
S_{s t r}=\frac{T_{20, r e f}}{T_{20, p a r}}
$$

where $T_{20, p a r}$ is the wall time spent for the speedup test and $T_{20, r e f}$ the wall time for the reference case. Subscript 20 indicates the problem size being fixed at 20 million. In the strong scaling test, the reference case is run using 64 CPU cores. Two PRACE (Partnership For Advanced Computing 
in Europe) HPC systems are tested, HERMIT in Germany and CURIE (FN) in France. Both show encouraging performances from the flow solver. They also suggest that there still are possibilities for further improvement, especially on the CURIE (FN) system the margin between ideal and actual is still large when more than 512 cores are used. The sampling approach for searching the minimum rounds may also be further improved with a more sophisticated optimization algorithm.

In Figures 11a and b, there is considerable difference between the ideal and actual speedup. This is a well known phenomenon in parallel CFD solvers. When the problem size (mesh size) is fixed, the ratio of the inter-partition facet number to the interior element number increases as the partition number increases. This means for each partition the communication overheads compared to the numerical computation within each partition (or each CPU core) are growing too. As a result, the actual strong scaling will gradually slow down and eventually flatten.

For the weak scalability, the mesh size is varying accordingly with the specified CPU cores. The used mesh size is between 5 and 50 million cells. The relative speedup for the week scaling is defined as:

$$
S_{w k}=\frac{N \times T_{5, r e f}}{T_{5 N, p a r}}
$$

where the reference case has a mesh size of 5 million and is performed using 64 CPU cores. $N$ is the ratio of number cores used in the speedup test against 64 . Notice the subscript denoting the problem size increases proportionally to the number of CPU cores. The performance in Figure 11c shows an excellent actual weak scalability, which is even slightly higher than "ideal". (Note: since the problem size is changing, it is possible that actual exceeds ideal).

It is also worth noting that although only marginal speedup gain is obtained in the present study in devising a user specified scheduling at the solver source code level, the approach's generic nature means it is applicable to similar flow solvers and is anticipated to be further improved by considering non-equal numbers of communication facets and the use of weighted graphs. Perhaps, more importantly it entirely avoids the need for non-blocking message passing giving further code robustness for large scale simulations.

\section{Concluding remarks}

This paper reports on successful computations of subsonic turbulent jets from a serrated nozzle in relation to a baseline axisymmetric nozzle. The results show good agreement with measurement data from a number of well documented experiments for the axisymmetric jet and the NASA Glen 
SHJAR experiments. The aim is to validate the numerical solution methods and hence to assess the sensibility of the flow solver due to changes made to the nozzle geometry (serration). For the baseline nozzle (SMC000), computed centerline mean velocities, spreading rate and Reynolds normal stress $\overline{u^{\prime} u^{\prime}}$ are similar to those measured at different Mach and Reynolds numbers. Similarity observed in incompressible jets is also evident in the current subsonic case. Second moments of velocity profiles across the jet shear layer agree favorably with with the data by Panchapakesan and Lumley. The computed two-point correlations without time delay on the centerline at $x=16 D$ indicates a well captured turbulent flow state as also presented in Bridges and Wernet's experiment.

Jet characteristics have also been explored for the serrated nozzle (SMC006) at the same unheated, high subsonic flow conditions. In contrast to the baseline jet, a much shortened potential core (circa 3.5 jet diameters) is captured from the numerical solution in excellent agreement with NASA Glen's SHJAR experiments. Also presented along the centerline is the normal Reynolds stress $\overline{u^{\prime} u^{\prime}}$. Despite the lack of smoothness to some extent, the profile peaks at the same location and reaches the similar maximum level as the measurement. Notable differences are observed along the centerline between two nozzles' solutions, indicating the good sensibility of the numerical methods and flow solver. The half-velocity jet width aimed to assess the jet spreading reveals that chevron effects are more pronounced up to the end of the potential core, after which the jet spreads more like an axisymmetric jet. The resolved two-point space-time cross correlations potentially with high values for acoustic modeling resemble typical Gaussian profiles giving further credibility to the presented numerical solutions. The instantaneous near field is examined by means of flow visualization. In particular, the vorticity contours comparison made for both jets reveal the fundamentally different mechanisms of shear layer growth in the near nozzle area.

The study also presents the work on parallel flow solver development on an improved scalability due to the incorporation of a user-level scheduling. Instead of letting the low-level MPI API functions to organize message passing, the scheduling takes the full control of communications sequence among different grid partitions and CPUs. This entirely removes the need for nonblocking sending and receiving operations, and it does not need or rely on extra buffer memory for non-blocking communications. 


\section{Acknowledgments}

The author would like to acknowledge the European PRACE systems for providing computing resources for code testing and development. Original experimental data obtained from NASA Glenn Research Center is gratefully acknowledged. The author also thanks the University of Sussex ITS department for the local HPC support and the use of the Apollo cluster.

\section{Nomenclature}

$\begin{array}{ll}a & \text { Speed of sound } \\ B_{u} & \text { Decaying slope constant } \\ D & \text { Nozzle diameter at jet exit } \\ d & \text { Wall distance } \\ \tilde{d} & \text { Modified wall distance } \\ d_{c} & \text { Starting distance of the mixed zone } \\ \mathbf{F} & \text { Flux vector } \\ f(\phi), g(\phi) & \text { Functions of } \phi \\ \mathbf{n} & \text { Outward normal unit vector } \\ p & \text { Pressure } \\ \mathbf{Q} & \text { Conservative variables } \\ R_{i j} & \text { Two-point velocity correlation }(i, j=1,2,3) \\ r & \text { Radius } \\ r_{0.5} & \text { Half-velocity jet width or radius } \\ S & \text { Relative speedup } \\ T & \text { Temperature; wall time } \\ t & \text { Physical time } \\ U_{c} & \text { Axial mean velocity on the jet centerline } \\ U_{j} & \text { Axial mean velocity at the nozzle exit } \\ u^{\prime}, v^{\prime}, w^{\prime} & \text { Velocity fluctuations in } x, r, \theta \text { directions } \\ u_{r m s}^{\prime} & \text { Cartesian coordinates } \\ \mathbf{v} & \mathbf{x}=(x, y, z)\end{array}$


Greek symbols

$\Delta$

$\epsilon$

$\epsilon_{0}$

$\epsilon_{1}$

$\tau$

$\theta$

$\Omega$

\section{Subscripts}

$a c$

$c$

$\infty$

j

$L$

$R$

0

Superscripts

$+$
Spatial separation

Small number

Laplacian coefficient

Damping coefficient

Time delay; pseudo time

Azimuthal angle

Vorticity

Acoustic values

On the jet centerline

Ambient values

At the jet exit

Immediate left of the interface

Immediate right of the interface

Jet virtual origin; Base point

\section{References}

[1] J. Banke. NASA helps create a more silent night. http://www.nasa.gov/topics/aeronautics/features/ bridges_chevron_events_prt.htm, December 2010.

[2] D. Bodony and S. K. Lele. Current status of jet noise predictions using large-eddy simulation. AIAA J., 46:364-380., 2008.

[3] J. Bridges and C. Brown. Parametric testing of chevrons on single flow hot jets. NASA/TM 2004-213107, 2004.

[4] J. Bridges and M. Wernet. Measurements of the aeroacoustic sound source in hot jets. NASA/TM 2004-212508, 2004.

[5] J. Bridges and M. Wernet. The NASA subsonic jet particle image velocimetry (PIV) dataset. NASA/TM 2011-216807, 2011. 
[6] T. Bui. A parallel, finite-volume algorithm for large-eddy simulation of turbulent flows. NASA/TM 1999-206570, 1999.

[7] M. Ciardi, P. Sagaut, M. Klein, and W. N. Dawes. A dynamic finite volume scheme for large-eddy simulation on unstructured grids. J. Comp. Phys., 210:632-655, 2005.

[8] P. A. Davidson. Turbulence: an introduction for scientists and engineers. Oxford University Press, Oxford, 2004.

[9] Arakeri V. H., A. Krothapalli, V. Siddavaram, M. B. Alkislar, and L. M. Lourenco. On the use of microjets to suppress turbulence in a Mach 0.9 axisymmetric jet. J. Fluid Mech., 490:75-98, 2003.

[10] J. O. Hinze. Turbulence. McGraw-Hill, 1975.

[11] H. J. Hussein, S. P. Capp, and W. K. George. Velocity measurements in a high-Reynolds-number, momentumconserving, axisymmetric, turbulent jet. J. Fluid Mech., 258:31-75, 1994.

[12] G. Karypis and V. Kumar. A fast and highly quality multilevel scheme for partitioning irregular graphs. SIAM J. Sci. Comput., 20(1):359-392, 1999.

[13] J. C. Lau, P. J. Morris, and M. Fisher. Measurements in subsonic and supersonic free jets using a laser velocimeter. J. Fluid Mech., 93:1-27, 1979.

[14] M. J. Lighthill. On sound generated aerodynamically. I. General theory. Proc. R. Soc. Lond. A, 211(1107):564$587,1952$.

[15] M. J. Lighthill. On sound generated aerodynamically. II. Turbulence as a source of sound. Proc. R. Soc. Lond. A, 222(1148):1-32, 1954.

[16] I. Mary and P. Sagaut. Large eddy simulation of flow around an airfoil near stall. AIAA J., 40:1139-1145, 2002.

[17] A. S. Monin and A. M. Yaglom. Statistical Fluid Mechanics, Mechanics of Turbulence, volume 1. MIT Press, Cambridge MA., 1971.

[18] C. J. Moore. The role of shear-layer instability waves in jet exhaust noise. J. Fluid Mech., 80:321-367, 1977.

[19] N. R. Panchapakesan and J. L. Lumley. Turbulece measurements in axisymmetric jets of air and helium. Part I. Air jet. J. Fluid Mech., 246:197-223, 1993.

[20] W. Rodi. A review of experimental data of uniform density free turbulent boundary layers. In B. E. Launder, editor, Studies in Convection. Academic, 1971.

[21] P. L. Roe. Approximate Riemann solvers, parameter vectors and difference schemes. J. Comp. Phys., 43:357-372, 1981.

[22] N. Saiyed. Separate flow nozzle test status meeting. NASA/TM 1997-210524, 1997.

[23] N. Saiyed, K. L. Mikkelsen, and J. Bridges. Acoustics and thrust of separate-flow exhaust nozzles with mixing devices for high-bypass-ratio engines. NASA/TM 2000-209948, 2000.

[24] M. L. Shur, P. R. Spalart, and M. K Strelets. Noise prediction for increasingly complex jets, Part II: Applications. Int. J. Aeroacous., 4:247-266, 2005.

[25] M. L. Shur, P. R. Spalart, M. K. Strelets, and Travin A. K. Towards the prediction of noise from jet engines. Int. J. Heat Fluid Flow, 24:551-561, 2003.

[26] A. Silberschatz, P. B. Galvin, and G. Gagne. Operating System Concepts. John Wiley \& Sons (Asia), 8th edition, 2010.

[27] P. R. Spalart and S. R. Allmaras. A one-equation turbulence model for aerodynamic flows. Recherche Aerospa- 
tiale, (1):5-21, 1994.

[28] Christopher K. W. Tam and Laurent Auriault. Jet mixing noise from fine-scale turbulence. AIAA J., 37:145-153, 1999.

[29] H. K. Tanna. An experimental study of jet noise: Part I turbulent mixing noise. J. Sound Vib., 50:405-428, 1977.

[30] A. A. Townsend. The Sturcture of Turbulent Shear Flows. Cambridge University Press, 2nd edition, 1976.

[31] P. G. Tucker. Novel MILES computations for jet flows and noise. Int. J. Heat Fluid Flow, 25:625-635, 2004.

[32] P. G. Tucker and L. Davidson. Zonal $k-l$ based large eddy simulations. Comput. Fluids, 33:267-287, 2003.

[33] P. G. Tucker, C. L. Rumsey, P. R. Spalart, R. E. Bartels, and R. T. Biedron. Computation of wall distances based on differential equations. AIAA Journal, 43(3), 2005.

[34] A. Uzun and M. Y. Hussaini. Simulation of noise generation in the near-nozzle region of a chevron nozzle jet. AAIA J., 47:1793-1810, 2009.

[35] B. van Leer. Towards the ultimate conservative difference scheme, V. a second order sequel to Godunov's method. J. Comp. Phys., 32:101-136, 1979.

[36] H. Xia and P. G. Tucker. Finite volume distance field and its application to medial axis transforms. Int. J. Numer. Methods Engng., 82:114-134, 2010.

[37] H. Xia and P. G. Tucker. Numerical simulation of single-stream jets from a serrated nozzle. Flow Turbul. Combust., 88:3-18, 2012.

[38] H. Xia, P. G. Tucker, and S. Eastwood. Large-eddy simulations of chevron jet flows with noise predictions. Int. J. Heat Fluid Flow, 30:1067-1079, 2009. 